\title{
Analysis of FS Stiffness Characteristics in Harmonic Gear Drive with CTC Tooth Profile
}

\author{
ZHENG Ji-Gui ${ }^{1, a^{*}}$, LIU Xu-Liang ${ }^{1, b}$, ZHANG He ${ }^{1, c}$, SHI Wei ${ }^{1, d}$ and ZHAO \\ Tian-Zhu ${ }^{1, e}$ \\ ${ }^{1}$ Beijing Research Institute of Precise Mechatronics and Controls, Beijing, 100076, China \\ azhengjigui@163.com, 'bivencool8@126.com, 'zh710@aliyun.com, dnjshiwei@163.com, ${ }^{\mathrm{e}} 1871413$ \\ 005@163.com, *Corresponding author
}

Keywords: CTC tooth profile; Harmonic drive; Analysis of stiffness characteristics

\begin{abstract}
Deforming process of FS (flexible spine) tooth is studied, its bending deformation and shear deformation are analyzed, and stiffness expression is concluded when FS tooth meshes into completely. FS cylinder torsional stiffness is analyzed with theoretical calculation and finite element analysis, the results of these two methods are compared, and reasons leading to different results are found out, which provides methods for analysis of stiffness characteristic in FS gear drive.
\end{abstract}

\section{Introduction}

Harmonic gear drive mechanism is one of gear drive mechanism, has some unique advantages, such as smooth movement, high transmission precision, large transmission ratio and high transmission efficiency, can transmit power and movement into confined space[1], and is used in more and more fields.

Harmonic gear drive is usually composed of three main parts, CS(Circular Spine), FS and wave generator. FS is the intermediary agency, and transmit power and torque through deformation[2]. FS stiffness characteristics have great effect on overall performance of harmonic reducer, for FS and CS both are rigid bodies. Based on the analysis of FS tooth deformation, its bending deformation and shear deformation are studied, and stiffness expression is concluded when the FS gear meshes into completely [3-5]. The FS barrel torsional stiffness is analyzed with theoretical calculation and finite element analysis, results of these two methods are compared, and reasons leading to different results are find out, which provides methods for analysis of FS tooth stiffness characteristics[6, 7].

\section{Analysis Of FS Tooth Deformation}

FS teeth will have flexible deformation on the surface and the whole body when on load, and its regions under stress in the different meshing position are different, stress situation at meshing beginning is as shown in Fig.1(a) and complete meshing situation is as shown in Fig.1(b). Tooth stiffness in these two cases above is obtained, whose average is considered as mean stiffness of each tooth. Tooth deformation can be divided into bending deformation and shear deformation, which can be solved separately.

When FS isn't in distortion, most teeth are in edge contact. Teeth's deformation is very complicate, and so circular-arc tooth profile can be simplified as a trapezoid whose upper base is addendum arc length, lower base is dedendum arc length and height is tooth height, as is shown in Fig1. 


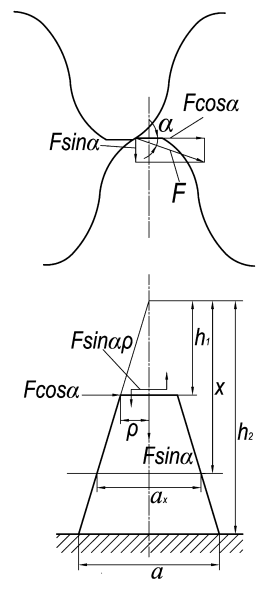

(a) Meshing beginning
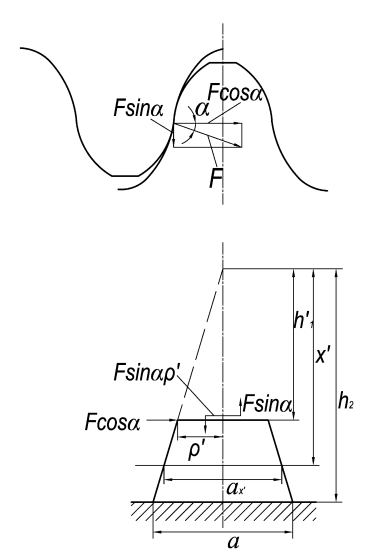

Fig1. Simplified force diagram of teeth corresponding to different meshing positions

Tooth bending deformation:

$$
y_{w}=\int \frac{\left(M_{1(x)}+M_{2(x)}\right) d x}{E I_{x}} \times \frac{\partial\left(M_{1(x)}+M_{2(x)}\right)}{\partial p}
$$

Where, $M_{1(x)}$ is bending moment variable produced by $F \cos \alpha, M_{1(x)}=-F \cos \alpha\left(x-h_{1}\right)$;

$M_{2(x)}$ is bending moment variable produced by $F \cos \alpha, M_{2(x)}=F \sin \alpha \rho$;

$I_{x}$ is polar moment variable of inertia $I_{x}=\frac{a^{3} x^{3}}{12 h_{2}^{3}}$.

$y_{w}=\frac{12 h_{2}^{3}}{E a^{3}} F \cos \alpha\left[\ln \frac{h_{2}}{h_{1}}+\frac{\left(h_{2}-h_{1}\right)\left(h_{1}-3 h_{2}\right)}{2 h_{2}^{2}}\right]+\frac{12 h_{2}^{3}}{E a^{3}} F \sin \alpha \rho \frac{-h_{1}\left(h_{1}-h_{2}\right)^{2}}{2 h_{1}^{2} h_{2}^{2}}$

Tooth shear deformation:

$y_{\tau}=\int \frac{k F \cos \alpha}{G m} d x$

Where, $k$ is constant with $1.2 ; m$ is basal area, in unit tooth width $m=\frac{a x}{h_{2}}$;

$G$ is shear elasticity modulus.

$y_{\tau}=\frac{k F \cos \alpha h_{2}}{G a} \ln \frac{h_{2}}{h_{1}}$

Linear elastic stiffness in unit tooth length:

$K_{0}=\frac{F \cos \alpha}{y_{w}+y_{\tau}}$

Tooth torsional stiffness:

$K_{1}^{\prime}=\frac{b K_{0} r}{\arcsin \frac{1}{r}} \times \frac{180}{\pi}$

Where, $b$ is tooth width; $r$ is FS radius in force point; $Z_{1}$ is number of teeth.

$$
K_{1}^{\prime}=\frac{180 b r}{\pi \arcsin \frac{1}{r}} \times \frac{1}{\frac{12 h_{2}^{3}}{E a^{3}}\left[\ln \frac{h_{2}}{h_{1}}+\frac{\left(h_{2}-h_{1}\right)\left(h_{1}-3 h_{2}\right)}{2 h_{2}^{2}}+\tan \alpha \rho \frac{-h_{1}\left(h_{1}-h_{2}\right)^{2}}{2 h_{1}^{2} h_{2}^{2}}\right]+\frac{k h_{2}}{G a} \ln \frac{h_{2}}{h_{1}}}
$$

Tooth stiffness is obtained when FS has completely meshed into.

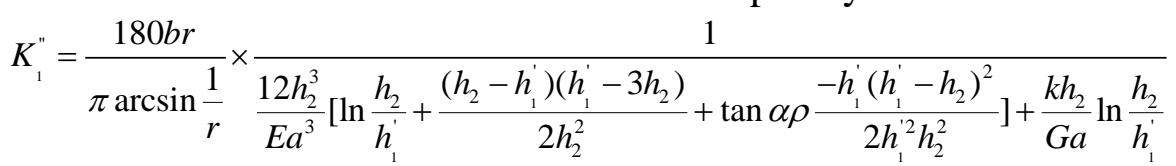

Mean stiffness of each tooth:

$K_{1}^{*}=\left(K_{1}^{\prime}+K_{1}^{\prime \prime}\right) / 2$ 
If meshing teeth number is $Z_{1} / 4$, then whole teeth stiffness is $K_{1}=Z_{1} K_{1}^{*} / 4$.

Tooth interface deformation is proportional to load, deformation changes with changing of tooth width and pressure angle. When other parameters are constant, tooth stiffness is proportional to tooth width $b$, the larger tooth width is, the smaller gear tooth radial deformation is, the bigger stiffness is, the bigger meshing pressure angle $\gamma$ is. While when tooth linear elastic stiffness is convert into torsional stiffness, FS radius increases, and torsional stiffness increases correspondingly.

\section{Torsional Stiffness Of FS Cylinder}

Structure of FS Cylinder. As is shown in Fig. 2, FS is composed of tooth width $b$, tooth I, outside FS cylinder II and flange connecting output shafts. Wall thickness of cylinder is composed of gear ring thickness $\delta_{1}$ and smooth cylindrical wall thickness $\delta$, and to simplify calculation, $\delta_{1}$ is taken the same value to $\delta$. To calculate torsional stiffness of cylinder, the cylinder can be decomposed into thin-wall cylinder II and flange connecting output shafts III, so torsional angle of cylinder $\theta$ is composed of thin-wall cylinder torsional deformation $\theta_{1}$ and flange torsional deformation connecting output shafts $\theta_{2}$.

Ttorsional deformation can be obtained from thin-wall structure of thin-wall cylinder with membrane analogy theory in Elasticity

$$
\theta_{1}=\frac{T l}{2 G \pi \delta(r+\delta / 2)^{3}}
$$

where, $T$ is troque; $G$ is shear elastic modulus; $r$ is radius of cylinder's inner wall; $\delta$ is wall-thickness; $l$ is cylinder length;

Torsional deformation angel of flange: $\theta_{2}=\frac{32 T D}{G \pi\left(r_{2}^{4}-r_{1}^{4}\right)}$.

Where, $r_{2}$ is outer diameter of flange; $r_{1}$ is inner diameter of flange; $D$ is thickness of flange.

Torsional stiffness of cylinder:

$$
K_{2}=\frac{T}{\theta_{1}+\theta_{2}}=\frac{1}{\frac{l}{2 G \pi \delta(r+\delta / 2)^{3}}+\frac{32 D}{G \pi\left(r_{2}^{4}-r_{1}^{4}\right)}}
$$

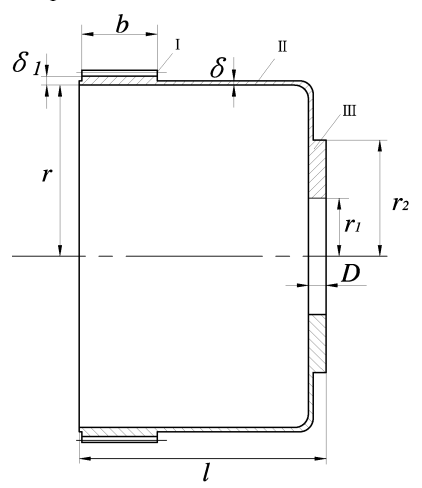

Fig.2 Schematic diagram of FS structure

Analysis Of Theoretical FS Stiffness. Calculation formula of FS integral stiffness:

$$
K=\frac{K_{1} K_{2}}{K_{1}+K_{2}}
$$

It can be concluded from the formula above midline of FS thin-wall section increases, so does cylinder stiffness. If cylinder length increases, then cylinder stiffness decreases. Cylinder wall-thickness increases, so does cylinder stiffness. Cylinder length, FS wall-thickness, flange-thickness, outer diameter of flange, and inner diameter of flange are changed separately, corresponding cylinder trosional stiffness is calculated, and relation curve is obtained, as is shown in Fig.3-7. Take the case for example, modulus $m=0.8$, teeth number $z_{1}=100$, pressure angel $\alpha=20^{\circ}$, tooth width $B=17 \mathrm{~mm}$, cylinder wall-thickness $\delta=0.7 \mathrm{~mm}$, cylinder length $l=55 \mathrm{~mm}$, outer diameter of 
flange $r_{2}=27 \mathrm{~mm}$, inner diameter of flange $r_{1}=17 \mathrm{~mm}$, flange-thickness $D=4 \mathrm{~mm}$. Theoretical FS torsional stiffness is $2.63 \times 10^{8} \mathrm{~N} \cdot \mathrm{mm} / \mathrm{rad}$, theoretical tooth stiffness is $5.02 \times 10^{10} \mathrm{~N} \cdot \mathrm{mm} / \mathrm{rad}$. Tooth flexibility accounts for $0.52 \%$ of FS integral flexibility. So it's safe to say that tooth's stiffness is much bigger than FS's, especially when there are more meshing teeth. In harmonic drive mechanism with many FS and CS teeth, teeth stiffness can be ignored.

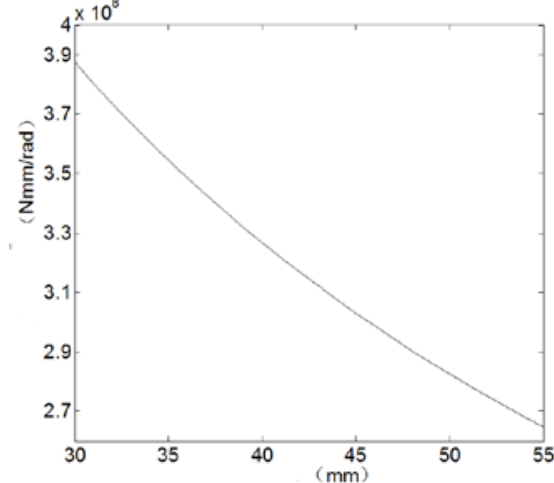

Fig.3 Relationship between FS torsional stiffness and cylinder length

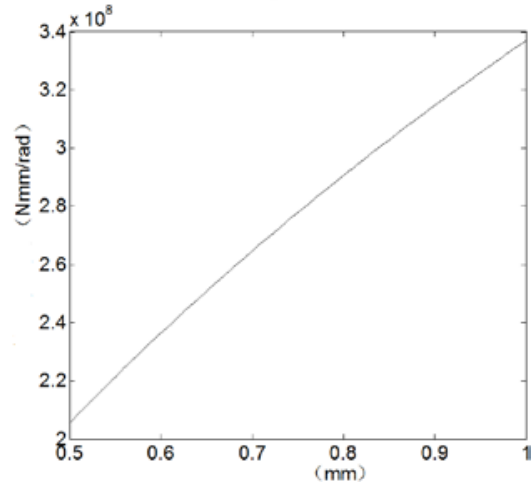

Fig.4 Relationship between FS torsional stiffness and FS wall-thickness

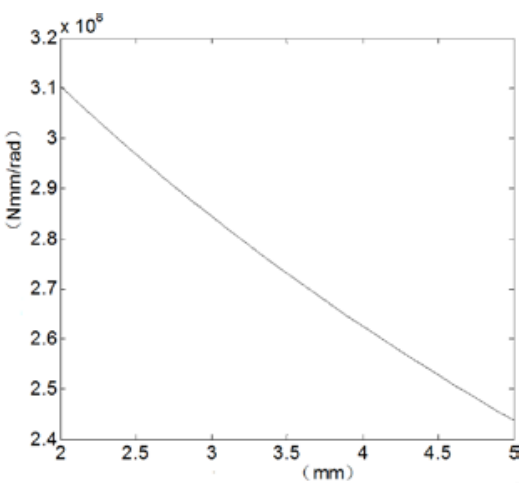

Fig.5 Relationship between FS torsional stiffness and flange-thickness

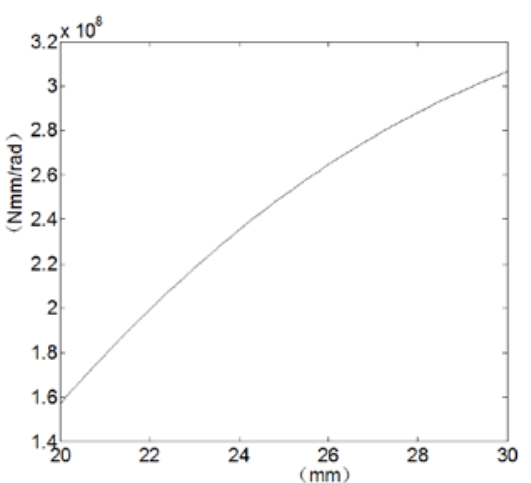

Fig.6 Relationship between FS torsional stiffness and outer diameter of the flange

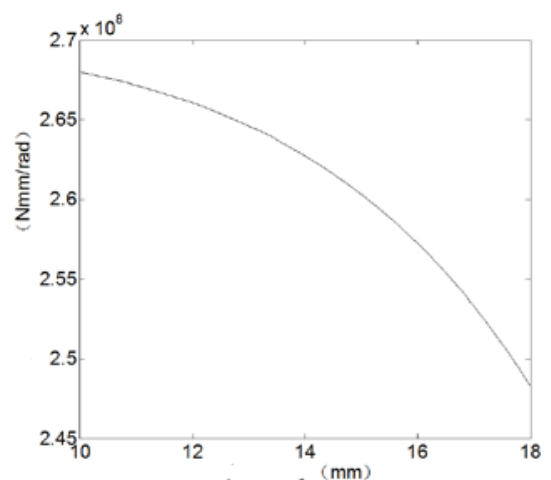

Fig.7 Relationship between FS torsional stiffness and inner diameter of flange

Analysis Of Teeth Meshing Contact. Meshing process of FS and CS is analyzed with finite element method, meshing teeth are always between initial meshing and complete meshing. Because tooth width is comparatively wide, stress is expressed with unit tooth length.
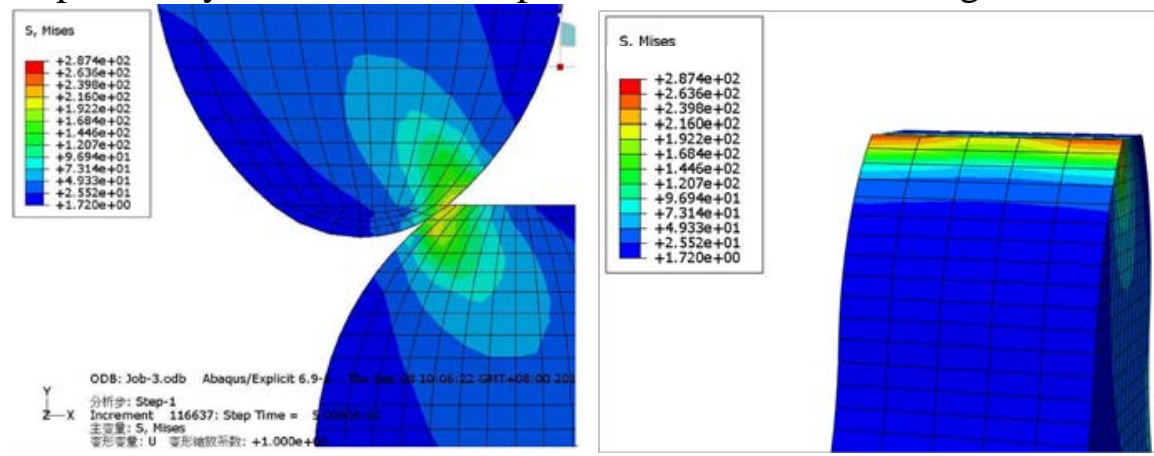

Fig.8 Mises stress nephogram in tooth initial meshing 

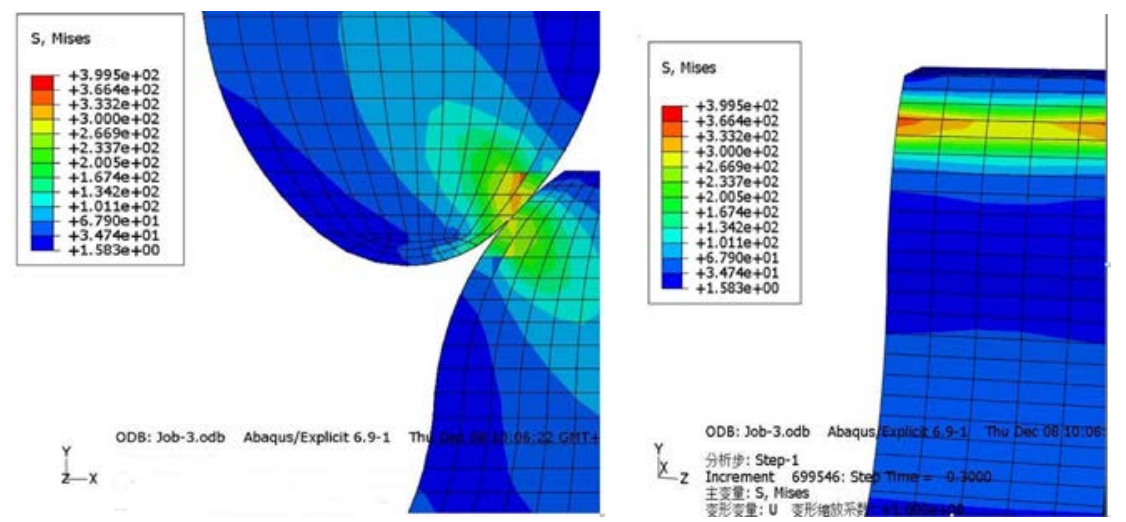

Fig.9 Mises stress nephogram when FS gear teeth in the greatest stress
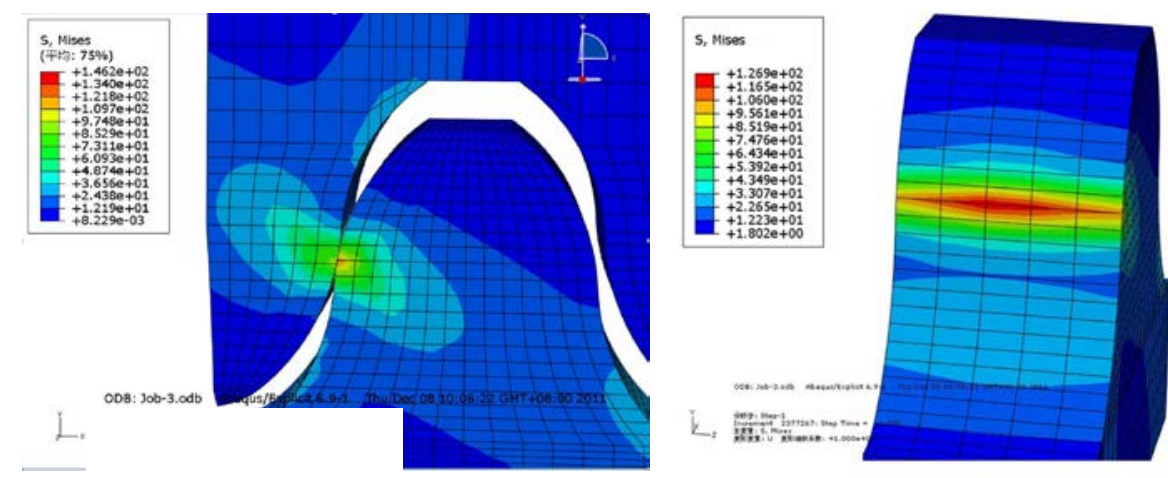

Fig.10 Mises stress nephogram when teeth in complete meshing

It can be conclude from Fig 8-10 that stress is quite big when FS teeth starts to mesh into CS, and after quite a short time stress arrives at the maximum, when bending moment imposed on tooth is the greatest, so is the stress; stress is quite small at the initial meshing, for stress on the other teeth hasn't been transmitted onto this tooth, and stress is at the minimum when tooth at its complete meshing.
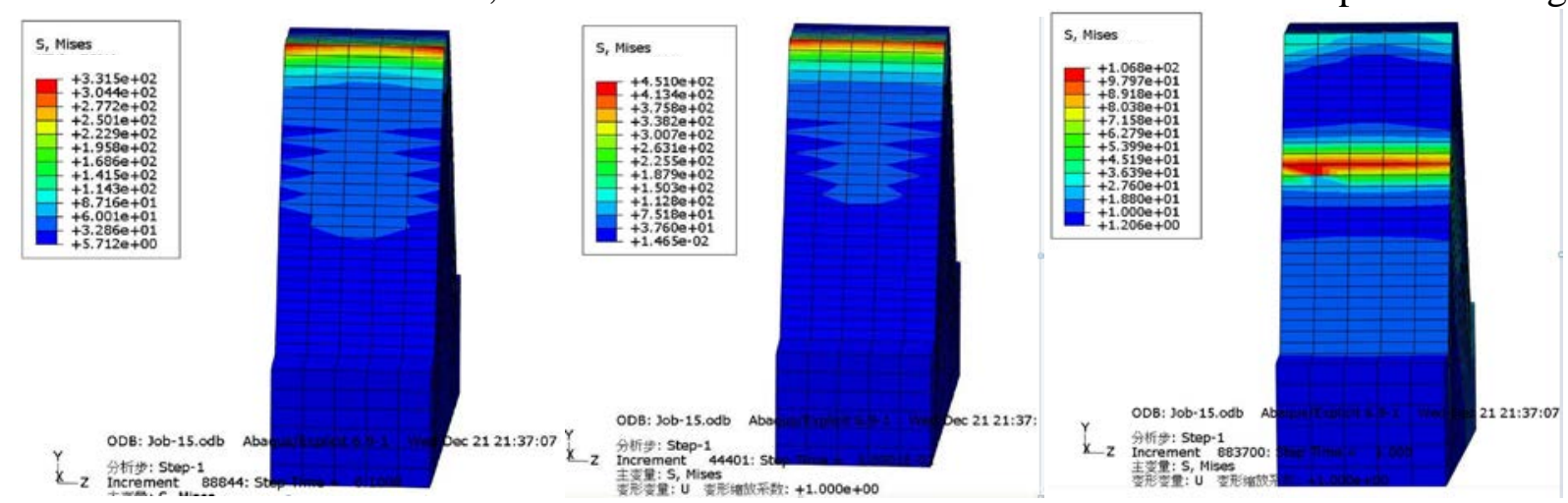

Fig.11 Mises stress nephogram of involute tooth profile meshing

For comparison, in analyzing involute tooth stress load imposed on FS is equal to that in analyzing circular-arc tooth. Mises stress nephograms of involute tooth starting to mesh into CS, tooth at its biggest stress, and tooth at its completely meshing into CS are shown respectively in Fig.11(a), (b) and(c). 


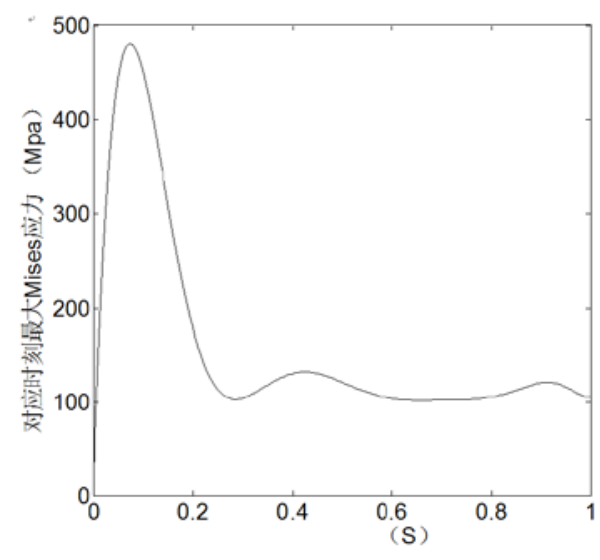

Fig 12. Mises biggest stress curve of involute tooth profile

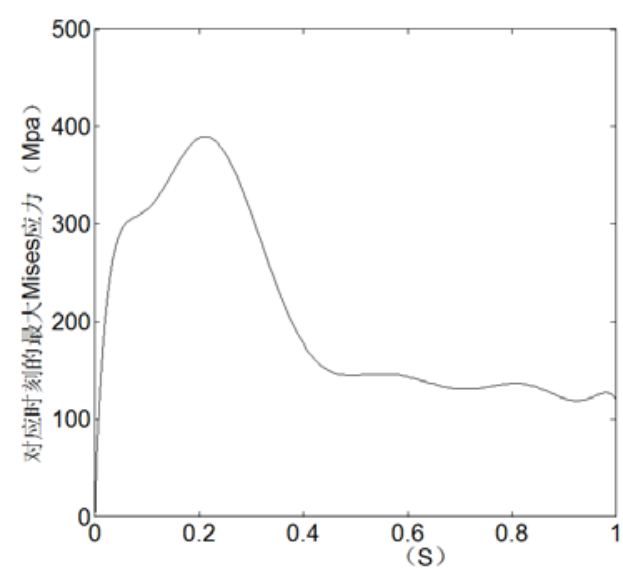

Fig 13. Mises biggest stress curve of circular-arc tooth profile

As is shown, stress change in tooth meshing process is the same as that in circular- arc tooth, value of which gets bigger at first and smaller later. Stress value is compared of circular-arc tooth and involute tooth at the same load at meshing beginning. At complete meshing stress of involute tooth is smaller than that of circular- arc tooth. Because in involute tooth there will be multiple line contact when FS in flexible deformation due to its bigger radius of curvature.

3.4 Infinite analysis of FS stiffness

Expression of FS cylinder and tooth stiffness concluded from above can be verified with finite element analysis. In finite element simulation CS and wave generator are fixed, FS output is imposed with torque, its load increases from $0 \mathrm{~N} \cdot \mathrm{m}$ to $100 \mathrm{~N} \cdot \mathrm{m}$ in $0 \mathrm{~S}-0.5 \mathrm{~S}$. The displacement of a pitch point on the cylinder is drew with load changing(the pinch point is on the FS flange), cylinder torsional stiffness is obtained with calculating its circumferential displacement, which is then compared with its theoretical value.

Displacement of pitch points on the X axis and Y axis direction is shown as in Fig.14 (a) and (b). The relationship between torque imposed on FS and its torque deformation is shown as in Fig15.

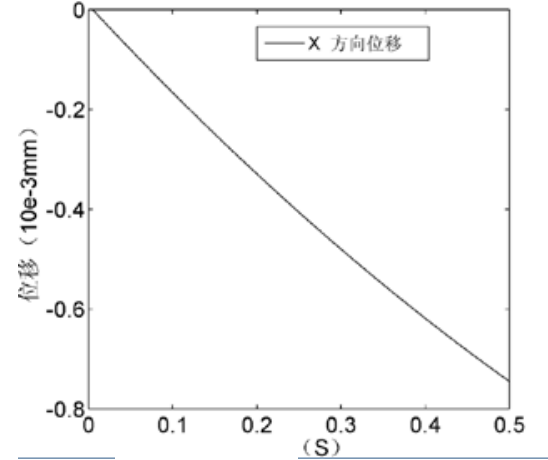

(a)

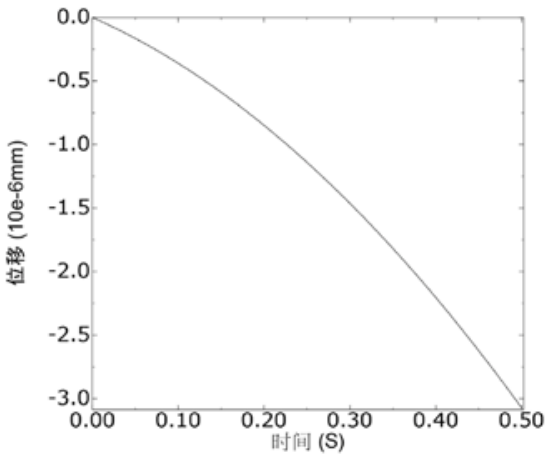

(b)

Fig.14 Displacement curve of pitch point

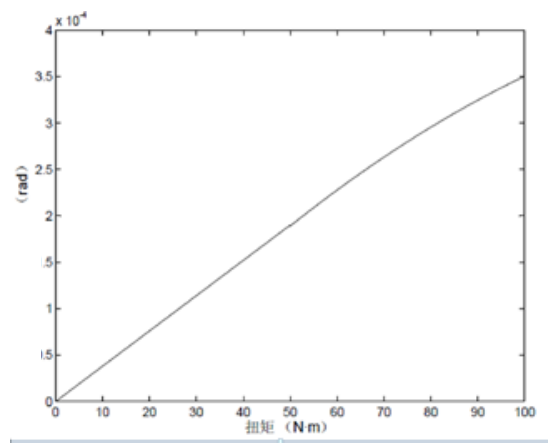

Fig. 15 Relation schema of torque and FS torional angle 
As is shown in Fig.15, when torque increases to $50 \mathrm{~N} \cdot \mathrm{m}$, slope of the curve gets smaller in "hysteresis" state. Not a single value characteristic of stiffness curve is due to teeth sliding friction loss in the harmonic gear tooth meshing and friction loss in wave generator, and the effect of material damping in finite element analysis when FS deforming; before torque increases to $50 \mathrm{~N} \cdot \mathrm{m}$, torque imposed on FS is about proportional to its torsional angle, slope of straight line is FS torsional flexibility(reciprocal of torsional stiffness). Result of FS stiffness finite element analysis can be obtained intuitively $2.49 \times 10^{8} \mathrm{~N} \cdot \mathrm{mm} / \mathrm{rad}$, which is a little smaller than the theoretical one $2.6834 \times 10^{8} \mathrm{~N} \cdot \mathrm{mm} / \mathrm{rad}$. To simplify the calculation fillet surface between thin-wall cylinder and disc hasn’t been calculated, so its result is a little bigger than that from finite element analysis.

\section{Conclusion}

FS tooth deformation is studied, its bending deformation and shear deformation are analyzed, and stiffness expression is concluded when FS teeth mesh into completely. FS cylinder torsional stiffness is analyzed, results from theoretical calculation and finite element analysis are compared, which provides methods for analysis of FS stiffness characteristic.

\section{References}

[1]Yunwen Shen. Theory and design in harmonic gear drive [M]. China Machine Press, 1985.

[2]Yougong Fan, Binghe Technical manual of harmonic gear drive [M]. National Defence Industry Press. 1995.

[3]Zhanzu Guo. Study on friction of harmonic gear reducer [J]. Journal of the Chinese academy of sciences changchun optical precision machinery and physics research institute, 2000.

[4]Weirong Ye. Optimization of meshing parameters in harmonic gear drive [D]. Harbin Institute of Technology, 2007

[5]Aidong Wang. Motion accuracy analysis of harmonic gear transmission mechanisim in robot [D]. Changchun Institute of Optics,Fine Mehcanics and Physics, Chinese Academy of Sciences,2001

[6]Guanghua Pan. Application of harmonic gear mechanism in business forms printing machine [J]. Packaging Engineering, 2003, 3 (24) :41.

[7]Furun Zhang, Boxun Luo. B matrix method of building meshing equation [J]. Journal of Huazhong University of Science and Technology, 1990, 2 (18). 